\title{
Histology-validated x-ray tomography for imaging human coronary arteries
}

Marzia Buscema, Georg Schulz, Hans Deyhle, Anna Khimchenko, Sofiya Matviykiv, et al.

Marzia Buscema, Georg Schulz, Hans Deyhle, Anna Khimchenko, Sofiya Matviykiv, Margaret N. Holme, Alexander Hipp, Felix Beckmann, Till Saxer, Katarzyna Michaud, Bert Müller, "Histology-validated x-ray tomography for imaging human coronary arteries," Proc. SPIE 9967, Developments in X-Ray Tomography X, 996700 (3 October 2016); doi: 10.1117/12.2238702

EDIE Event: SPIE Optical Engineering + Applications, 2016, San Diego, California, United States 


\title{
Histology-validated X-ray tomography for imaging human coronary arteries
}

\author{
Marzia Buscema*a, Georg Schulz ${ }^{\mathrm{a}}$, Hans Deyhle ${ }^{\mathrm{a}}$, Anna Kimchenko ${ }^{\mathrm{a}}$, Sofiya Matviykiva ${ }^{\mathrm{a}}$, Margaret \\ N Holme ${ }^{b}$, Alexander Hipp ${ }^{c}$, Felix Beckmann ${ }^{c}$, Till Saxer ${ }^{\mathrm{d}}$, Katarzyna Michaud ${ }^{\mathrm{e}}$, \\ and Bert Müller ${ }^{\mathrm{a}}$ \\ aBiomaterials Science Center, University of Basel, Gewerbestrasse 14, 4123 Allschwil, Switzerland; \\ ${ }^{\mathrm{b}}$ Department of Materials, Imperial College London, United Kingdom; ${ }^{\mathrm{c}}$ Institute of Materials \\ Research, Helmholtz-Zentrum Geesthacht, 21502 Geesthacht, Germany; ${ }^{d}$ University Hospitals of \\ Geneva, 1206 Geneva, Switzerland; 'University Center of Legal Medicine, University of Lausanne, \\ Lausanne, Switzerland
}

\begin{abstract}
Heart disease is the number one cause of death worldwide. To improve therapy and patient outcome, the knowledge of anatomical changes in terms of lumen morphology and tissue composition of constricted arteries is crucial for designing a localized drug delivery to treat atherosclerosis disease. Traditional tissue characterization by histology is a pivotal tool, although it brings disadvantages such as vessel morphology modification during decalcification and slicing. X-ray tomography in absorption and phase contrast modes yields a deep understanding in blood vessel anatomy in healthy and diseased stages: measurements in absorption mode make visible highly absorbing tissue components including cholesterol plaques, whereas phase contrast tomography gains better contrast of the soft tissue components such as vessel walls. Established synchrotron radiation-based micro-CT techniques ensure high performance in terms of 3D visualization of highly absorbing and soft tissues.
\end{abstract}

Keywords: Phase contrast, single grating interferometry, double grating interferometry, soft and calcified tissue visualization, three-dimensional rendering, lumen segmentation, atherosclerosis.

\section{INTRODUCTION}

Cardiovascular diseases (CVDs) are a group of disorders of the cardiovascular system including the heart and blood vessels. They can lead to severe pathologies such as cerebrovascular disease, peripheral arterial disease and coronary heart disease, namely atherosclerosis. Atherosclerosis is an inflammatory reaction caused by the accumulation of white blood cells (WBCs) in the inner walls of the arteries. This accumulation leads to deposit of fats, cholesterol, calcium, platelets and cellular debris making the arteries thick and hard [1]. The narrowing of the blood vessel reduces the blood flow, thus decreasing oxygen supply to the heart and leads to heart insufficiency. When an atherosclerotic plaque ruptures a drastic decreases in the blood flow occurs leading to heart attack or stroke. Tobacco use, unhealthy diet and obesity, physical inactivity and excessive use of alcohol are among the main risk factors of heart attack and stroke. It is known that almost $50-60 \%$ of death due to stroke and heart attack occurs within the first hour. In the ambulance, one of the conventional protocols to treat stroke and heart attack is intravenous injection of a vasodilator, such as nitroglycerine together with high concentration of oxygen inhalation and a platelet activity inhibitor such as acetylsalicylic acid. Oxygen delivery decrease the workload on the heart and vasodilation increases the local perfusion pressure both limiting infarction damage. A systemic vasodilation, however, leads to the decrease of peripheral vascular resistance with a consequent fall in blood pressure. A local release of vasodilators is a solution to this drawback [2]. As the constriction of the artery leads to a significant increase in the arterial flow velocity, it creates a difference in shear stress between healthy and constricted area [3]. Indeed, one can take advantage of the differences in the blood velocity to release the drug locally, using a purely physical trigger [4].

\footnotetext{
*marzia.buscema@unibas.ch; phone +41 61 2075438; bmc.unibas.ch
}

Developments in X-Ray Tomography X, edited by Stuart R. Stock, Bert Müller, Ge Wang, Proc. of SPIE Vol. 9967, 996700 · C 2016 SPIE · CCC code: 0277-786X/16/\$18 · doi: 10.1117/12.2238702 
Recently, a shear-stress sensitive nano-container formulation was discovered [5]. Figure 1 shows a schematic representation of the liposome and the related chemical structure. The novelty of this artificial phospholipid-based liposome lies in the lenticular shape: the equator of such liposomes likely represents an easier breaking point under mechanical stimuli because of the reduced order of phospholipids. Each artery occlusion and wall shear stress within every patient is distinctive, due to variations in plaque thickness, lesion length and blood viscosity. Therefore, one of the crucial steps for the successful delivery of vasodilators is a better understanding of the local shear stress changes at the site of the occlusion. This information later can be used for blood flow simulations [6]. Depending on the shear values, molecular structure and mechanical properties of the liposomes have to be properly tailored. To monitor liposome properties, a promising approach is to combine small-angle X-ray scattering (SAXS) and microfluidics [7]. A deep knowledge of the artery morphology, including both plaque structure and lumen area, has to be obtained. Histological examination has been used for centuries, and is considered as a gold standard for tissue characterization. It reaches submicrometer resolution in two dimensions, but is limited in the third one due to slice thickness and limited slices number. Histological sample preparation of the atherosclerotic artery is a critical step, since the cross-sectional area of the lumen can be modified during cutting and decalcifying giving inappropriate results for further flow simulation analysis. Moreover, histology belongs to the destructive analysis methods. In order to overcome these difficulties, non-destructive three dimensional (3D) X-ray imaging, such as micro computed tomography $(\mu \mathrm{CT})$, is promising alternative for the characterization of atherosclerotic coronary arteries $[5,6,8-10]$. One of the problems of X-ray tomography in absorption mode is the simultaneous visualization of soft and highly absorbing tissues: If the photon energy is optimized for the visualization of highly absorbing tissues, the soft tissues are almost transparent to the X-rays and provide poor contrast. If it is optimized for soft tissues, artifacts arise due to the high absorption in the calcified tissue. However, one can take advantage of phase contrast mode, which is less sensitive to variations in electron density and atomic number of the constituents and leads to improved soft tissue contrast. Nevertheless, both techniques together with histology are complementary [6]. In this study, data from a calcified human coronary artery have been acquired in absorption contrast mode using the laboratory system nanotom ${ }^{\circledR} \mathrm{m}$ (Phoenix|x-ray, GE Sensing \& Inspection Technologies GmbH, Wunstorf, Germany) to identify plaque- containing areas. To simultaneously visualize both highly absorbing and low absorbing tissue, phase contrast synchrotron radiation based $\mu \mathrm{CT}$ tomography (SR $\mu \mathrm{CT})$ measurements using grating interferometry have been carried out. The data were acquired using both single and double grating setups. SR $\mu C T$ grating interferometer-based measurements usually required two gratings: one of the gratings, namely beam-splitter, produces an interference pattern; the other one, namely analyzer grating, is used to analyze the pattern from the first grating. The absorption grating can however be removed if the beam-splitter grating period is large enough so that the detector can resolve the interference pattern directly.

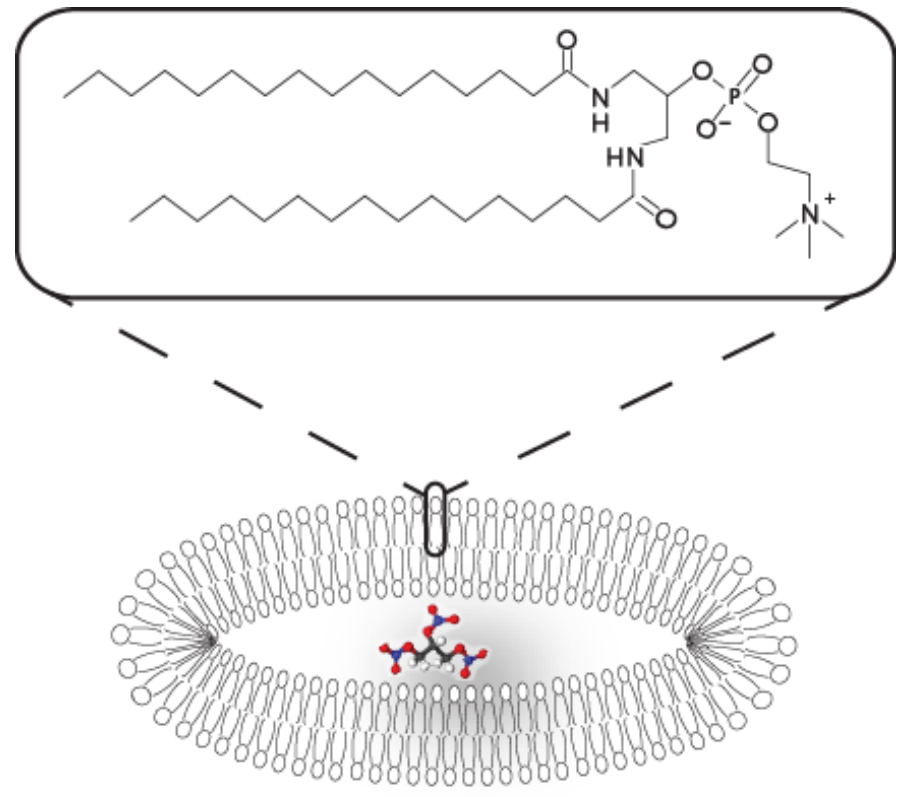

Figure 1. Schematic showing the non-spherical structure of the artificial phospholipid-based liposome loaded with nitroglycerine. The chemical structure of the liposome is given. 


\section{MATERIALS AND METHODS}

\subsection{Sample preparation}

A portion of a calcified human coronary artery $2.2 \mathrm{~cm}$-long, severed from the distal part of the anterior interventricular artery, was explanted post mortem from a 73-year old woman who donated her body for research purposes to the Plateforme d'enseignement de morphologie Faculté de biologie et de médecine, Département de neurosciences fondamentales, University of Lausanne, Switzerland. The surrounding tissue, mainly fatty tissue, was removed from the artery by using a scalpel. The sample was placed in a $2.5 \mathrm{~mL}$ Eppendorf tube filled with $4 \%$ paraformaldehyde (PFA). To avoid air bubble formation during X-ray exposition, the sample was left in a shaking plate for at least $12 \mathrm{~h}$ before imaging.

\subsection{Absorption contrast mode at the laboratory $\mu \mathrm{CT}$ system}

The calcified human coronary artery was first visualized with a nanotom ${ }^{\circledR} \mathrm{m}($ Phoenix $\mid \mathrm{x}$-ray, GE Sensing \& Inspection Technologies $\mathrm{GmbH}$, Wunstorf, Germany) operating in absorption contrast mode. The machine, is equipped with a 180 $\mathrm{kVp} / 15 \mathrm{~W}$ nanofocus tube with a $\mathrm{W}$ target $[11,12]$. Measurements were performed at an accelerating voltage of $60 \mathrm{kVp}$ and a beam current of $310 \mu \mathrm{A}$. The Eppendorf tube was vertically positioned on a high precision stage and rotated through the X-ray cone beam. 1400 equiangular projections were taken over $360^{\circ}$. For each projection angle, nine images were recorded with a total exposure time of $4.5 \mathrm{~s}$. A pixel length of $10.4 \mu \mathrm{m}$ was used. The data were reconstructed with the software datos|x 2.0 (Phoenix|x-ray, GE Sensing \& Inspection Technologies GmbH, Wunstorf, Germany).

\subsection{X-ray grating interferometer-based phase tomography at the synchrotron source}

The measurements in grating- based phase contrast mode were carried out at the beamline P07 (Helmhotz-Zentrum Geesthacht, DESY, Hamburg, Germany). The sample was placed in a water tank to reduce the related artifacts. Both configurations in single grating and double grating were used in order to find the best setup and parameters for simultaneous visualization of highly absorbing and soft tissues. The measurement was performed with a double-crystal Si monochromator with a bent crystal to enhance the flux but also to enlarge the bandwidth. The optics consisted of a $100 \mu \mathrm{m}$-thick $\mathrm{CdWO}_{4}$ scintillator, a $5 \times$ magnifying optics and a CMOS-Camera. The camera was developed and produced by Karlsruhe Institute of Technology (KIT/IPE) and HZG and is based on a 20MPix CMOSIS-chip with a native pixel size of $6.4 \mu \mathrm{m}$.

Single grating setup: A photon energy of $45 \mathrm{keV}$ has been set. In this configuration, the beam-splitter grating $(10 \mu \mathrm{m}$ periodicity) was placed at $80 \mathrm{~cm}$ from the detector, corresponding to around the first Talbot distance. For each projection angle, four phase stepping images were acquired over one period of the interference pattern. The effective pixel size was $1.3 \mu \mathrm{m} .900$ off-axes projections were taken over $360^{\circ}$ with an exposure time of $0.2 \mathrm{~s}$ per each phase step.

Double grating setup: The sample was measured at a mean photon energies of $40 \mathrm{keV}$ and $45 \mathrm{keV}$. The beam-splitter grating $(4.8 \mu \mathrm{m}$ periodicity, $\mathrm{Ni})$ was placed $27.9 \mathrm{~cm}$ from the analyzer grating $(2.4 \mu \mathrm{m}$ periodicity, $\mathrm{Au})$ in the configuration at $40 \mathrm{keV}$. At the photon energy of $45 \mathrm{keV}$, the first grating was placed $31.4 \mathrm{~cm}$ from the second one, corresponding to the third Talbot distance. The effective pixel size was $1.3 \mu \mathrm{m}$, the number of 900 off-axes projections over $360^{\circ}$, and the exposure time $0.2 \mathrm{~s}$ per each phase step. For each projection angle, four phase stepping images were acquired over one period of the interference pattern.

The XGI-based phase tomography image shown in Figure 2 is part of a measurement carried out at the beamline ID19 (ESRF, Grenoble, France). Here, a photon energy of $53 \mathrm{keV}$ was selected using a double-crystal Si (111) monochromator. The sample was placed $11.5 \mathrm{~cm}$ from the beam-splitting grating ( $4.8 \mu \mathrm{m}$ periodicity, $11 \mu \mathrm{m}$-thick Ni) whereas the analyzer grating $(2.4 \mu \mathrm{m}$ periodicity, $100 \mu \mathrm{m}$-thick $\mathrm{Au})$ was at a distance of $36.7 \mathrm{~cm}$ from the beam-splitter grating and $10.5 \mathrm{~cm}$ from the detector. The X-ray detector unit included a $\mathrm{CdWO}_{4}$ luminescent screen, optical lens and CCD camera with a pixel size of $7.5 \mu \mathrm{m} .999$ projections were acquired over $360^{\circ}$ with an exposure time of $2 \mathrm{~s}$ per phase step. For each projection angle, four phase-stepping images were acquired over one period of the interface pattern. More details about grating interferometry and the phase stepping method can be found in Weitkamp et al. [13]. The effective pixel size corresponded to $5.18 \mu \mathrm{m}$ with a resulting field of view of $4.7 \mathrm{~mm} \times 10.6 \mathrm{~mm}$. 


\subsection{Data registration}

Datasets acquired in single and double grating configurations, were registered using an automatic $3 \mathrm{D} / 3 \mathrm{D}$ registration tool $[14,15]$ with rigid transformation constrains. The dataset acquired in double grating setup using a photon energy of 45 $\mathrm{keV}$ was used as reference.

\subsection{Histology}

The calcified human coronary artery was immersed in decalcifier (distilled water: formic acid: PFA, 87: 8: $5 \mathrm{v} / \mathrm{v}$ ) at a temperature of $37^{\circ} \mathrm{C}$ for two days. Subsequently, it was dehydrated by sequential soaking in alcohol then xylene overnight at reduced pressure (Tissue-Tek VIP E300 Histokinetic automated dehydrator, Sakura) and immersed in molten paraffin at a temperature of $60^{\circ} \mathrm{C}$. The paraffin was allowed to cool and the block was trimmed with a scalpel to the size of the artery-containing region. Histological sections of 2-4 $\mu \mathrm{m}$ thickness were cut (Microm Cool-cut HYRAX M 40, Carl Zeiss), stained with Hematoxylin \& Eosin (H\&E) stain and mounted on glass slides. Each slice was photographed using a Nikon Eclipse 80i microscope and camera at 10× magnification in an average of 20 sections and stitched together using the stitching plug-in [16] available in the Fiji software (ImageJ version 1.45k, EMBL, Germany) [17]. This gave color images of on average of $4500 \times 5500$ pixels $(0.5 \mu \mathrm{m} \times 0.5 \mu \mathrm{m})$ that were binned to reduce the size of the dataset for ease of handling, and converted to grayscale images for further analysis.

\section{RESULT AND DISCUSSION}

\subsection{Histology vs. X-ray tomography}

Histology is the gold standard for tissue characterization. However the preparation steps, including decalcification and slicing, modify drastically the morphology of the sample. Such modification includes changes in the lumen crosssectional area [18] making it difficult to perform precise flow simulations. Therefore, X-ray tomography techniques are a valuable tool providing nondestructive imaging of soft tissue avoiding some deformation of the sample morphology. Figure 2 shows the comparison between a histological slice of a decalcified human coronary artery (left) and the corresponding 2D slice obtained from X-ray tomography data in phase contrast mode that were carried out at the beamline ID19 (ESRF, Grenoble, France). Differences in the lumen cross-section can clearly be seen.
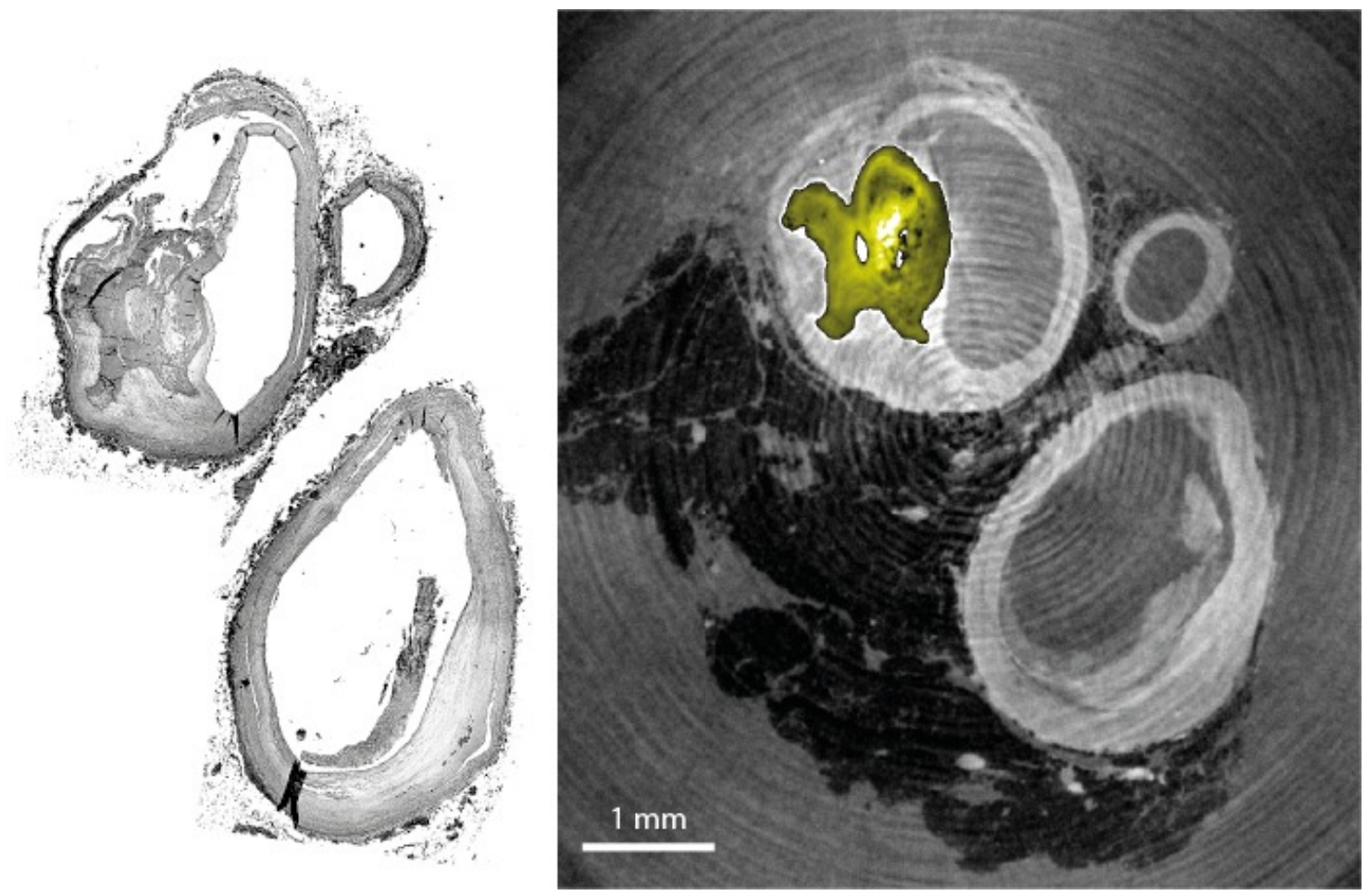

Figure 2. Decalcified histological section 2-4 $\mu$ m-thick of a human coronary artery (left) and the corresponding double grating phase contrast $\mathrm{SR} \mu \mathrm{CT} 2 \mathrm{D}$ image (right). 


\subsection{Absorption contrast for calcified tissue visualization using an advanced laboratory setup}

The calcified human coronary artery fixed in formalin was first measured in absorption contrast mode by means of an advanced laboratory $\mu \mathrm{CT}$ system. Figure 3 shows a 2D cross sectional slice of the artery. Here, the calcified tissue namely plaque - is clearly visible, whereas it is difficult to differentiate the soft tissues from the formalin.

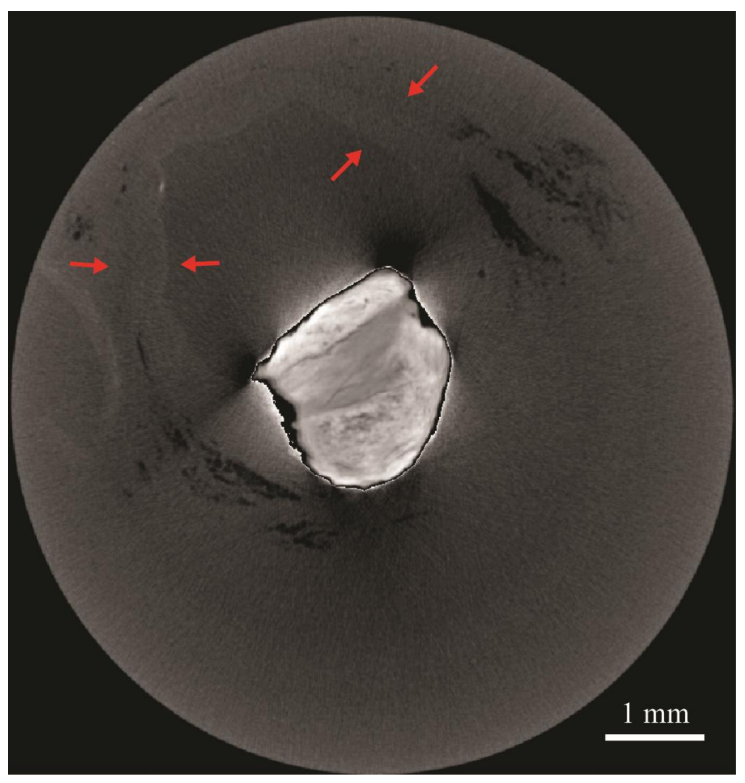

Figure 3. 2D slice taken from the dataset acquired in absorption contrast mode. The bright part is the calcification, namely plaque; the dark grey parts correspond to surrounding tissue, mainly fatty tissues. The light grey part marked by red arrows indicates the vessel walls.

\subsection{XGI-phase SR $\mu \mathrm{CT}$ : single vs. double grating configurations}

The visualization of the highly absorbing and soft tissue-containing human coronary artery by measuring in absorption contrast mode is challenging. To better discern among the tissue types within the same sample the XGI phase contrast modality was used. Figure 4 shows a comparison between single and double grating modalities. The double grating results show less artifacts and better differentiation of the soft tissues (light grey, dark dots) with respect to the calcification (yellow). This allows for an easier segmentation of the lumen - the area still available for the blood to flowto be used as sound basis for flow simulations [6]. Based on the calculation of beam separation formula $2 \lambda d / p$, the angular sensitivity of the system can be estimated, being $\lambda$ the beam energy, $d$ the distance from the detector (single grating setup) or the distance between the two gratings (double grating setup) and $p$ the beam-splitter grating periodicity [19]. It was found an angular sensitivity of $0.66 \mathrm{mrad}$ and $0.16 \mathrm{mrad}$ for double and single grating, respectively.
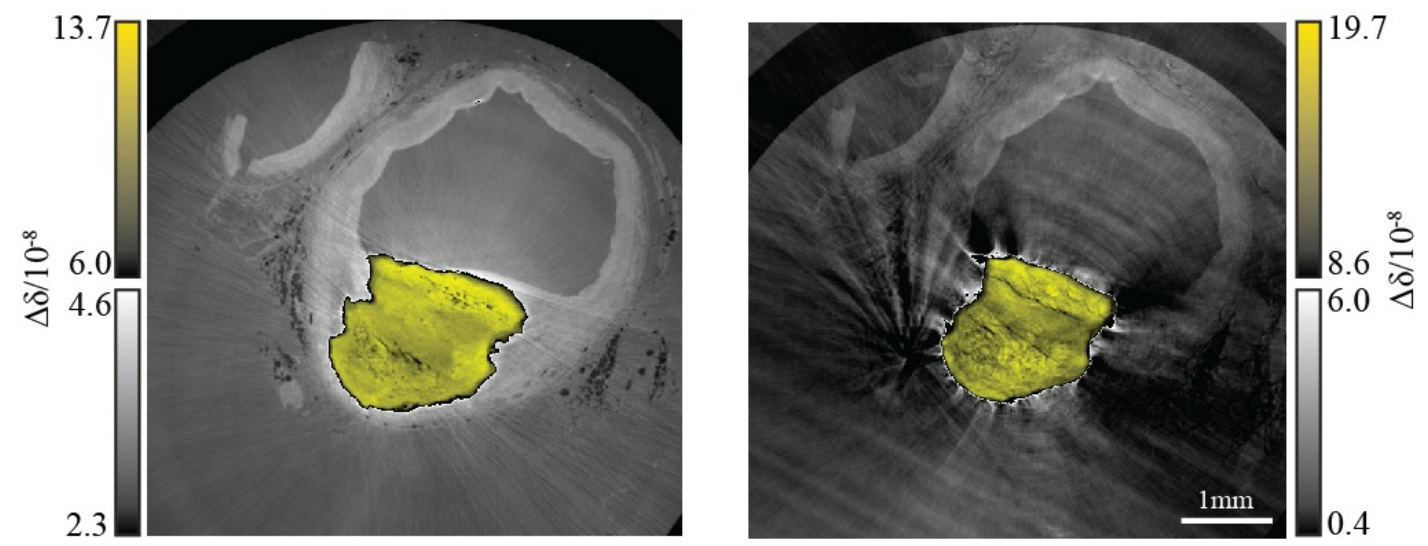

Figure 4. Qualitative comparison of 2D slices obtained in phase contrast mode by using double (left) and single grating (right) setup. To simultaneously visualize soft and calcified tissue grey and yellow scales were used. 


\subsection{Comparison of double grating phase contrast tomograms by using two photon energies}

For a simultaneous visualization of the vessel walls and the plaque of the human coronary artery the choice of the beam energy is not trivial. Figure 5 shows two cross sectional slices from the measurements carried out in double grating using $40 \mathrm{keV}$ (left) and $45 \mathrm{keV}$ (right). Both datasets were registered as reported above (cf. section 2.4). For both slices, a line was taken across the section of the artery (Fig.5, bottom): the two lines (in red for $40 \mathrm{keV}$ and in blue for $45 \mathrm{keV}$ ) show comparable results. However, for the $45 \mathrm{keV}$ data more details of the highly absorbing tissue are visible, as expected. In the $40 \mathrm{keV}$ data, the lower contrast for the fatty tissue (dark dots) can be related to the interpolation error.

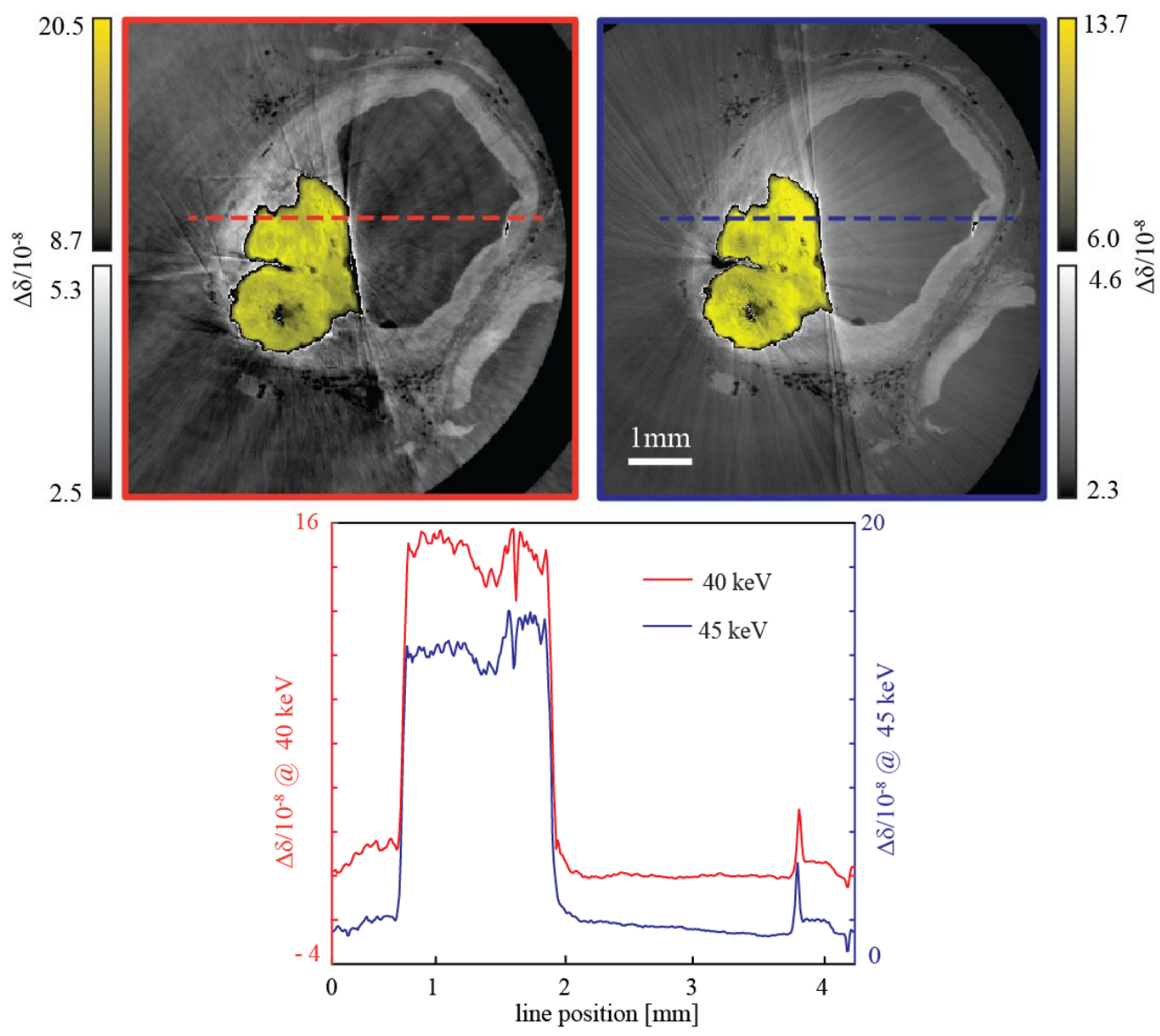

Figure 5. Comparison between 2D slices of a calcified human coronary artery measured at photon energies of $40 \mathrm{keV}$ (left) and $45 \mathrm{keV}$ (right). The calcification is shown in yellow, the vessel walls in light grey and the surrounding tissue in dark grey. The features present in the sample are shown in the plot (below) by taking a line across both the 2D cross sectional slices.

\subsection{Three dimensional visualization of a plaque-containing human coronary artery}

The quality of the data obtained from grating interferometry based measurements in phase contrast mode, allows to discriminate the tissues in a calcified human coronary artery [6]. Figure 6 shows the 3D images of a portion of the plaque-containing sample obtained from double grating measurements at $45 \mathrm{keV}$. After the intensity-based segmentation, the calcification can be easily visualized (Fig. 6, right) with respect to softer tissues (Fig. 6 left). The semitransparent rendering (Fig. 6, center) shows the location of the calcification within the artery.

\section{CONCLUSIONS}

To optimize shear-stress sensitive artificial phospholipid-based liposomes for a local release of vasodilators a deep understanding of the morphology of both healthy and constricted human coronary arteries is needed. Histology allows for tissues visualization down to the sub-micrometer scale. However, the slice preparation modifies the morphology of 
the sample as shown in Figure 2. In order to visualize the morphology and the tissue composition of human coronary arteries, X-ray tomography-based data using both laboratory X-ray source and synchrotron radiation source have been acquired on a calcified human coronary artery. To visualize and localize the calcified tissue, we firstly measured the specimen using a laboratory setup in absorption contrast mode. To better differentiate among the tissues, the sample was subsequently measured in phase contrast modality by using two grating interferometer-based geometries. Even an improved data acquisition results in data that are hardly segmentable using intensity-based implementation of commercially available software.
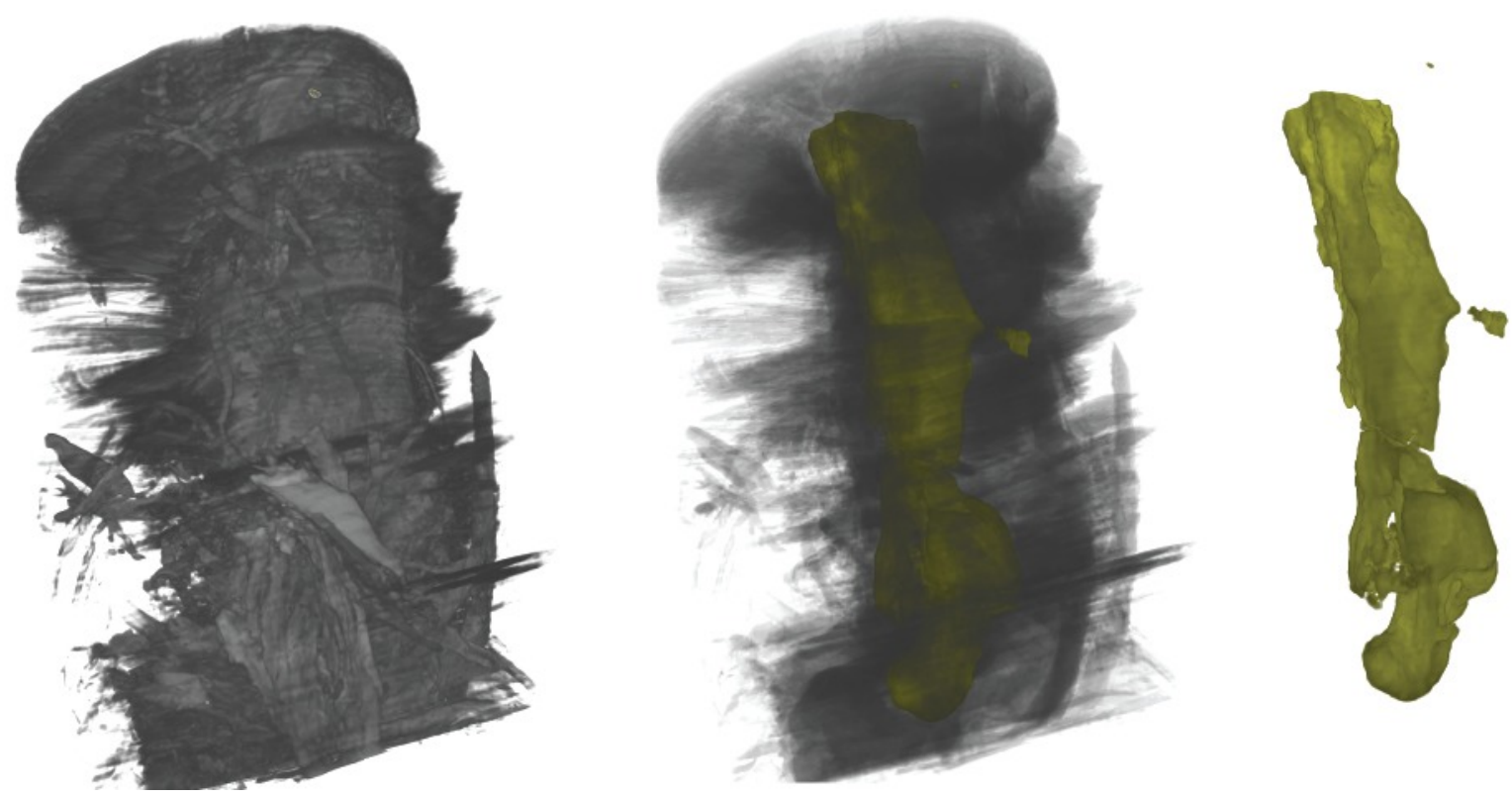

Figure 6. 3D visualization of a portion of a plaque-containing human coronary obtained from double grating dataset at 45 $\mathrm{keV}$. From left to right: outer rendering of the artery, semitransparent rendering and morphology of the plaque.

\section{ACKNOWLEDGMENT}

This work was partially funded by the Swiss National Science Foundation (SNSF) in the National Research Program (NRP) 62 'Smart Materials'. The authors thank University Center of Legal Medicine, University of Lausanne, Switzerland for providing the human artery. The SNSF R'Equip project 133802 is kindly acknowledged.

\section{REFERENCES}

[1] http://www.heart.org,

[2] Saxer, T., Zumbuehl, A., and Müller, B., "The use of shear stress for targeted drug delivery," Cardiovasc. Res. 99(2), 229-231(2013)

[3] Wellnhofer, E., Osman, J., Kertzscher, U., Affeld, K., Fleck, E., and Goubergrits, L., "Non-dimensional modeling in flow simulation studies of coronary arteries including side-branches: A novel diagnostic tool in coronary artery disease," Atherosclerosis 216(2), 277-282(2011)

[4] Korin, N., Kanapathipillai, M., Matthews, B. D., Crescente, M., Brill, A., Mammoto, T., Ghosh, K., Jurek, S., Bencherif, S. A., Bhatta, D., Coskun, A. U., Feldman, C. L., Wagner, D. D., and Ingber, D. E., "Shear-Activated Nanotherapeutics for Drug Targeting to Obstructed Blood Vessels," Science 337(6095), 738(2012)

[5] Holme, M. N., Schulz, G., Deyhle, H., Hieber, S. E., Weitkamp, T., Beckmann, F., Herzen, J., Lobrinus, J. A., Montecucco, F., Mach, F., Zumbuehl, A., Saxer, T., and Müller, B., "Morphology of atherosclerotic coronary arteries," Proc. SPIE 8506, 850609(2012) 
[6] Holme, M. N., Schulz, G., Deyhle, H., Weitkamp, T., Beckmann, F., Lobrinus, J. A., Rikhtegar, F., Kurtcuoglu, V., Zanette, I., Saxer, T., and Müller, B., "Complementary X-ray tomography techniques for histology-validated 3D imaging of soft and hard tissues using plaque-containing blood vessels as examples," Nat. Protoc. 9(6), 1401-1415(2014)

[7] Buscema M, Deyhle, H., Pfohl, T., Hieber, S. E., Zumbuehl, A., and and Müller, B., "Characterization of mechano-sensitive nano-containers for targeted vasodilation," Proc. SPIE 9797(97970S), (2016)

[8] Buscema, M., Holme, M. N., Deyhle, H., Schulz, G., Schmitz, R., Thalmann, P., Hieber, S. H., Chicherova, N., Cattin, P. C., Beckmann, F., Herzen, J., Weitkamp, T., Saxer, T., and B, a. M., "Grating interferometry-based phase microtomography of atherosclerotic human arteries," Proc. SPIE 9212, (2014)

[9] Khimchenko, A., Schulz, G., Deyhle, H., Hieber, S. E., Hasan, C., Bikis, C., Schulz, L., Costeur, L., and and Müller, B., "Non-destructive phase contrast hard X-ray imaging to reveal the three-dimensional microstructure of soft and hard tissues.," Proc. SPIE 97970B, (2016)

[10] Müller, B., Schulz, G., Deyhle, H., Stalder, A. K., Ilgenstein, B., Holme, M. N., Weitkamp, T., Beckmann, F., and Hieber, S. E., "X-ray microscopy of soft and hard human tissues," AIP Conference Proceedings 1696(0200101-4), (2016)

[11] Electric, G., " Measurement and Control, Phoenix nanotom m 180 kV / 20 W X-ray nanoCT system for highresolution analysis and 3D metrology," (2014)

[12] Egbert, A., and and Brunke, O., "High-resolution X-ray computed tomography for materials research," Adv. Mat. Res. 222, 48-51(2011)

[13] Weitkamp, T., Diaz, A., David, C., Pfeiffer, F., Stampanoni, M., Cloetens, P., and Ziegler, E., "X-ray phase imaging with a grating interferometer," Opt. Express 13(16), 6296-6304(2005)

[14] Andronache, A., von Siebenthal, M., Szekely, G., and and Cattin, P., "Non-rigid registration of multi-modal images using both mutual information and cross-correlation," Med. Image Anal 12(1), 3-15(2008)

[15] Müller, B., Deyhle, H., Lang, S., Schulz, G., Bormann, T., Fierz, F., and Hieber, S. E., "Three-dimensional registration of tomography data for quantification in biomaterials science," International Journal of Materials Research 103(2), 242-249(2012)

[16] Preibisch, S., Saalfeld, S., and Tomancak, P., "Globally optimal stitching of tiled 3D microscopic image acquisitions," Bioinformatics 25(11), 1463-1465(2009)

[17] Walter, T., Shattuck, D. W., Baldock, R., Bastin, M. E., Carpenter, A. E., Duce, S., Ellenberg, J., Fraser, A., Hamilton, N., Pieper, S., Ragan, M. A., Schneider, J. E., Tomancak, P., and Heriche, J.-K., "Visualization of image data from cells to organisms," Nat. Methods 7(6), 479-479(2010)

[18] Goderie, T. P., van Soest, G., Garcia-Garcia, H. M., and Gonzalo, N., "Combined optical coherence tomography and intravascular ultrasound radio frequency data analysis for plaque characterization. Classification accuracy of human coronary plaques in vitro," Int. J. Cardiovasc. Imaging 26, 843-850(2010)

[19] Modregger, P., Pinzer, B. R., Thuring, T., Rutishauser, S., David, C., and Stampanoni, M., "Sensitivity of X-ray grating interferometry," Optic Express 19(18324), (2011) 